\title{
Assessment of Channel Interference for Licensed Shared Access (LSA) in the 2.3-2.4 GHz band
}

\author{
Esko Luttinen \\ Oulu University, \\ Finland \\ Email: eskok.luttinen@elisanet.fi
}

\author{
Marja Matinmikko \\ VTT Technical Research Center of \\ Finland \\ Oulu, Finland \\ Email: marja.matinmikko@vtt.fi
}

\author{
Marcos Katz \\ Centre for Wireless Communications \\ Department of Communication \\ Engineering \\ University of Oulu \\ Email: marcos.katz@ee.oulu.fi
}

\begin{abstract}
This text simulates sharing conditions between incumbent spectrum user and mobile network operator (MNO) at the frequency band $2.3-2.4 \mathrm{GHz}$. The probability of interference between radio systems helps to determine appropriate frequency planning rules and specific limits for system performances. Due to rapid technology development in mobile devices and network technologies an explosive growth in mobile traffic demand has led to the need for more market driven approaches for spectrum access. European spectrum regulation administrations have started to consider the development of strategic approaches and policies for spectrum sharing under the Licensed Shared Access (LSA) framework. In Europe the 2.3-2.4 $\mathrm{GHz}$ band has been identified as the first potential application area for sharing between a MNO and incumbent spectrum users under LSA framework. Simulations are conducted to assess the sharing conditions between incumbent program making and special events (PMSE) services and entrant LTE (Long Term Evaluation) network. One difficulty in simulations is to describe occurancy of busy and idle periods of intended sharing systems.
\end{abstract}

Keywords-component; cognitive radio, interference management, simulation study, spectrum sharing

\section{INTRODUCTION}

Increasing mobile traffic demand will lead to increasing spectrum demand for mobile communication networks [1]. The availability of new exclusive spectrum for mobile network operators (MNOs) is restricted due to other incumbent spectrum usage in the frequency bands suitable for the mobile service. This has led to explore new innovative strategies and technologies to improve radio spectrum sharing. Spectrum sharing should exploit such technologies that the primary users spectrum bands are interference free [2]. Spectrum sharing is a potential means for making new spectrum available for MNOs. Spectrum sharing with rules and conditions that resemble exclusive licensing are preferable for MNOs in order to offer service level guarantee and controlled interference environments. Spectrum sharing is a framework, the development of which requires the involvement of all relevant stakeholders.

Applications of the spectrum sharing models to specific frequency bands have been studied including the TV white space case as a notable example. The utilization of cognitive radio systems for efficient sharing of TV white spectrum have been studied e.g. by COGEU in [3]. Authors in [4] considered the availability of TV white space spectrum in Europe for sharing. Modelling of spectrum availability for sharing based on measurement studies was studied in [5]. The Quasar project studied a quantitative assessment of secondary spectrum access [6]. Opportunistic sharing between a radar system and a cellular network was studied in [7]. Previous works have mainly considered the unlicensed sharing case using TV white spaces. Recently the Licensed Shared Access (LSA) concept has emerged in Europe for sharing under licensed conditions which is more attractive for MNOs. In Europe CEPT is developing harmonized conditions for the use of LSA in the 2.3 $-2.4 \mathrm{GHz}$ band [8]. In Europe there are several independent National Regulatory Authorities (NRAs), which require their own implementations for interference-free communication services. There is not much prior published research work on the assessment of spectrum usability for sharing in the 2.3-2.4 $\mathrm{GHz}$ band under the LSA concept. The aim of these studies was to provide generic guidelines to CEPT administrations of implementation of LSA concept. The cognitive radio trial study was done by the Core+ project/VTT Finland. The demonstration document of this study is described in [9].

Similar spectrum sharing developments are taking place outside Europe. In USA the PCAST report [10] indicates that in the coming years, access to spectrum will be an increasingly important foundation for America's economic growth and technological leadership. In US the study covers the technical requirements, architecture and operational parameters of the proposal Spectrum Access System (SAS) for the 3550 - 3650 $\mathrm{MHz}$ band. NTIA (National Telecommunications and Information Administration) looks to the 4G LTE industry to assist in analyzing the data and the results, and perhaps performing additional tests [11].

The aim of this paper is two-fold, to describe European system model of the LSA and secondly to simulate coexistence of low power LTE time division duplex (TDD) mode and cordless camera and to assess possibilities of interference free communication services of these systems at the low power and with the broadband spectrum masks in the $2.3-2.4 \mathrm{GHz}$ band.

The rest of this paper is organized as follows. The system model and problem formulation are presented in Section II including a description of the new LSA/ASA regulatory concept and the current use of the 2.3-2.4 GHz band in Europe. An analysis of the usability of spectrum for sharing based on the LSA concept in the mentioned band is then discussed by presenting the simulation environment and numerical simulations with selected results in Sections III. Conclusions are drawn in Section IV and acknowledgements in section V. 


\section{SYSTEM MODEL}

\section{A. Licensed Shared Access (LSA)}

A solution to facilitate spectrum sharing at $2.3-2.4 \mathrm{GHz}$ in Europe is depicted in the figure 1. A new regulatory approach "Licensed Shared Access (LSA)" has been proposed by the European Commission (EC) in [12]. EC has defined LSA as " $a$ regulatory approach aiming to facilitate the introduction of radio communication systems operated by a limited number of licensees under an individual licensing regime in a frequency band already assigned or expected to be assigned to one or more incumbent users. [13].

The LSA regulatory approach aims to protect the rights of incumbents, i.e., current holders of spectrum rights of use, while introducing additional access rights to the same band on licensed basis. It is particularly attractive for MNOs to share spectrum from other type of incumbent spectrum users, which corresponds to the recently introduced industry-driven Authorized Shared Access (ASA) concept presented in [14]. LSA and ASA could benefit from cognitive radio system (CRS) technology by identifying sharing opportunities that previously were considered unfeasible.

Currently, the LSA approach is being studied by the European regulation and standardization bodies with a particular application for the mobile broadband in the 2.3-2.4 $\mathrm{GHz}$ band in Europe. The European Conference of Postal and Telecommunications Administrations (CEPT) have established two project teams, FM52 and FM53, to address the LSA concept. FM53 has developed a regulatory framework for LSA and its application to mobile broadband in [15]. FM52 is studying the applicability of the LSA concept for mobile broadband specifically in the $2.3-2.4 \mathrm{GHz}$ band in Europe. The group is has developed an ECC Decision on harmonizing implementation measures for MFCN (e.g., broadband wireless access systems) including least restrictive technical conditions. It also considers border coordination issues. LSA related standardization activities are currently on-going in European Telecommunication Standards Institute (ETSI) in its Technical Committee on Reconfigurable Radio Systems (RRS) following a system reference document on LSA for mobile broadband in the 2.3-2.4 GHz band [16].

The current regulatory and standardization activities on LSA are specifically addressing the cases where MNOs would share spectrum from other type of incumbent spectrum users on bands allocated to the mobile service and identified for International Mobile Telecommunication (IMT) systems. This specifically corresponds to the ASA concept. From now on we use the term LSA throughout this paper. To reach a good balance between regulators, mobile network operators and incumbent spectrum users, there must be realistic willingness of co-operation. Also technology issues for implementing the sharing need to be solved and techno-economic studies have to be done in parallel with network operators. As LSA solutions are new there are many uncertainties with the input parameters of both technical and business calculations - a proposed way of business and technical calculations are done on a case-by-case basis.

\section{B. LTE}

LTE TDD has standardized already in 3GPP in release 8 and LTE TDD is a suitable solution for mobile broadband technology for both large-scale coverage deployment and applications such as broadcasting and small cells (i.e., femtoand pico-cells). LTE TDD asymmetry feature, where uplink and downlink data rates can be varied dynamically, matches different needs. The downlink rate can be up to nine times higher that the uplink rate by splitting the time durations correspondingly. LTE TDD can be considered as a regional or band-specific solution to spectrum access, mature technology for LTE small cells [17][18].

\section{C. $S A P / S A B$}

In Europe, temporary SAP/SAB video links are used in the frequency band $2.3-2.4 \mathrm{GHz}$ for a variety of purposes. Typical application scenarios and technical characteristics are described in ERC Report 38 [19]. Video link types include cordless cameras, portable links, mobile links and temporary point-to-point links. Cordless camera links consist of handheld cameras with portable receivers with maximum effective isotropic radiated power EIRP of $6 \mathrm{dBW}$. Application range of cordless cameras is less than $500 \mathrm{~m}$. The coexistence of broadband wireless system and Services Ancillary to Programme making/Services Ancillary to Broadcasting (SAP/SAB) video link has been studied in the ECC Report 172 [20] and results indicate that the required coupling loss depends on the video link scenario. According to [20] coexistence is feasible in adjacent or larger separation channel distances on case-by-cases depending on e.g., antenna directions, sometimes requiring additional protection.

\section{Spectrum sharing}

An MNO could expand its operation by accessing a shared spectrum band to increase MNO capacity for a certain location subscribers. The sharing devices should support also the shared spectrum. As the spectrum usability is dependent upon three arguments: time, place and frequency, we have the following choices:

- Space: If there were several access points in the LSA band, the frequency sharing users should know the places where the specific spectrum is available to use. Coordination of the use is essential between the incumbent and the MNO.

- Time: Typically incumbent spectrum users, such as TV companies, use cordless cameras temporarily, when making reports for news. The more densely populated areas, the more frequent usage could be expected. The LSA users need to know when and for how long the LSA band is available for sharing.

- Frequency: The LSA users also need to know which specific frequencies in the band are available for sharing. Long-term availability of spectrum for sharing in the foreseeable future is important to consider. In the international definitions the band $2.3-2.4 \mathrm{GHz}$ is the 3GPP TDD channel 40 [21] and its use for mobile communications is under study. 
If the regulatory body of a country (NRA) divides the spectrum by frequency range and geographic boundaries, unwanted emissions, receiver blockings, co-channel and adjacent channel intermodulation products should meet acceptable level of communication [22]. Planning rules require specifications limits for transmitters and receivers performances. A NRA participates to the sharing by granting licenses and controlling devices and system protocols. In spectrum sharing systems, the NRA has to define firstly how to divide the channel access time between the incumbent and the additional LSA licensee network users [23], and secondly, what is the spectrum rent level for the LSA licensee users. If the LSA licensee from time to time has to release the spectrum totally to its incumbent users, what will be the release time in abundance of the spectrum to primary users, and would there be an alternative communication band in transfer of the LSA licensees' communication? A framework and measurements are needed in coordination of the network parameters and enhancing performance to control both primary and secondary user's communication. If the primary network holders make all the decisions to maximize their usage the network resource is limited on the secondary user point of view. The situation is even more difficult, if there are several incumbent and LSA licensees using the shared network.

It is a challenge to define good performance metrics for all applications of sharing. For example video transmission is a spectrum hungry application. The application of MPEG video, voice over IP and other information sensitive media provides preliminary measurement of network capability. Quality of service (QoS) over packet switched networks needs to monitor the real time effectiveness of the network and ease of diagnose in a loss case, because video quality is a complex function of the network layer statistics [24]. The document [25] defines media delivery index (MDI), which is a set of measures to be used to monitor both the quality of a delivered video stream as well as to show system margin for IPTV systems. The measurements provide an accurate measurement of jitter and delay at network level.

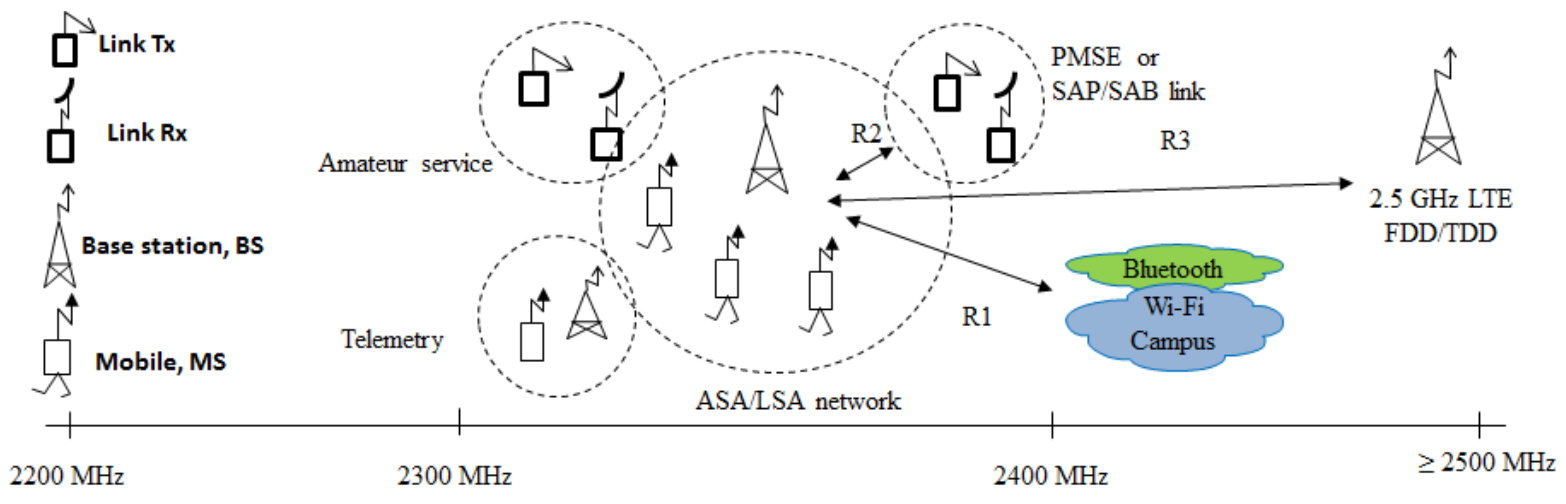

\begin{tabular}{|l|l|}
\hline$\leq \mathbf{2 . 3} \mathbf{~ G H z}$ freq. usage & Freq. usages between $\mathbf{2 . 3} \mathbf{- 2 . 4} \mathbf{~ G H z}$ \\
Defence Systems & Aeronautical Telemetry \\
Fixed Links & Amateur \\
Radio Astronomy & Mobile Applications \\
SAP/SAB & Defence Systems \\
EESS/Space,Operation/Space Research & Fixed Links \\
Mobile applications & Radio Astronomy \\
Space Research (deep space) & SAP/SAB \\
\hline
\end{tabular}

ERC25, a source document of frequency allocations for CEPT member countries in Europe.

In Finland the frequency range $2290-2315 \mathrm{MHz}$ : permission only for a case-to-case occasions. License duration only the time of the occasion

Figure 1 European networks in the 2.3-2.4 GHz frequency band, below and above [26].

In Europe it may be difficult to find a common agreement and frequency coordination between MFCN (Mobile Fixed Cellular Networks) and other incumbent systems at the band 2.3-2.4 $\mathrm{GHz}$, because there are 27 countries using the band for various applications, and because in many CEPT member countries there are multiple operators and radio systems (Fig.1). The European working groups FM52 and FM53 work for coordination and for bilateral and multilateral agreements [27]. The introduction of mobile communication networks at the band of 2.3-2.4 GHz requires also country based NRAs' implementations. The disruptive models of agreement may open applications for new players to offer connectivity services. For cooperating operators this requires accurate analysis of the economic benefits. Firstly one may ask on what level incumbents or MNOs are prepared to hire, share or use the available band. Secondly do current NRAs support releasing or sharing the band for commercial basis, and then is there a clear process for sharing. Typical co- and adjacent channel interference ranges between LTE TDD and SAP/SAB video link cases at $2-3-2.4 \mathrm{GHz}$ are listed in the document [20]. 
In mobile communication interference between two systems occurs, when systems share the same physical environment and their spectrum operation has overlapping frequencies at the time of communication [28] (see Fig. 2). The sharing studies should provide a method to define a tolerable limit for co-channel and adjacent channel interferences..

The total interference power $\mathrm{I}_{\text {total }}$ is a sum of background and receiver noise of the victim system $(\mathrm{N})$, interfering system interference $\left(\mathrm{I}_{1}\right)$ and additional interference $\left(\mathrm{I}_{2}\right)$, which is dependent on several interferences like number of active transmitters or distances between operating systems:

$$
\mathrm{I}_{\text {total }}=\mathrm{N}+\mathrm{I}_{1}+\mathrm{I}_{2} \text {. }
$$

The noise rise $(\mathrm{R})$ is defined as the ratio of total received power to the noise power of the system, we get the noise rise:

$$
\mathrm{R}=\mathrm{I}_{\text {total }} / \mathrm{N}=1 /(1-\eta) \text {. }
$$

By defining usability factor $(\eta)$ and rearranging we could get the victim systems usability in Eg. (3):

$$
\eta=1-\left(\mathrm{N} / \mathrm{I}_{\text {total }}\right)
$$

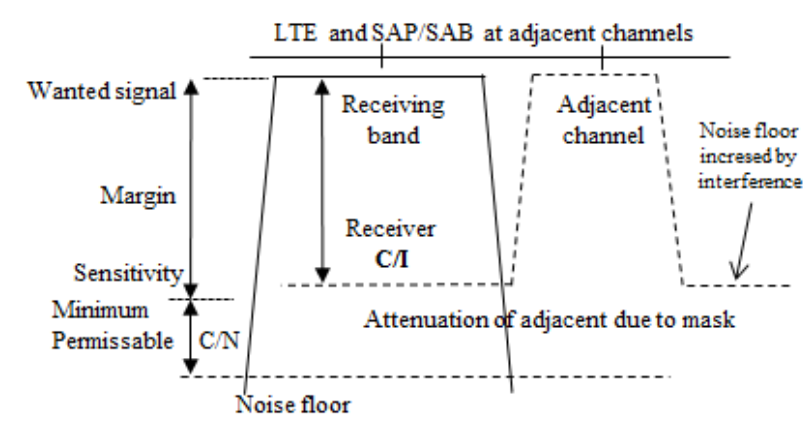

Figure 2 Interference on adjacent channel

The noise $(\mathrm{N})$ of the victim system is known and the total interference $\left(\mathrm{I}_{\text {total }}\right)$ can be measured. The efficiency of radio spectrum use can be simulated via compatibility studies between different radio systems and services to identify interference-free areas. The problem of unwanted emission and interference are serious factors affecting QoS of the communication. The level of interference should be expressed in terms of probability [29]. The statistical methodology, the well-known Monte-Carlo approach, has been used for simulating of random processes, where samples of random variables are taken from their defined probability density functions.

\section{E. Simulation tool}

SEAMCAT® (Spectrum Engineering Advanced MonteCarlo Analysis Tool) is the implementation of a radio simulation model developed by the group of CEPT Administrations [30] [31]. The SEAMCAT tool is used for sharing and compatibility studies between different radio systems operating in the same or adjacent frequency bands. The mathematic functions, Monte-Carlo radio simulation methodologies, are expressed in the document [29]. The SEAMCAT tool is simulation model developed by the group of CEPT Administrations. The tool is public object code software distributed by the CEPT European Radio communications Office (ERO). The SEAMCAT 4.1.0 version was used [30].
The Seamcat tool calculates received signal strength and unwanted and blocking interfering signals. In interference-free reception carrier to noise relation $(\mathrm{C} / \mathrm{N})$ indicates the victim receiver sensitivity level (Fig.2). The probability of interference with the Seamcat tool are: interference criterion $\mathrm{C} / \mathrm{I}, \mathrm{C} /(\mathrm{N}+\mathrm{I}),(\mathrm{N}+\mathrm{I}) / \mathrm{N}$ or $\mathrm{I} / \mathrm{N}$, where $\mathrm{C}$ indicates carrier, I interfering signal and $\mathrm{N}$ noise level. As $\mathrm{C} / \mathrm{I}$ was selected as interference criterion, for a proper connection carrier to interference ratio (C/I) must be greater than required $\mathrm{C} / \mathrm{I}$ threshold in order to avoid interference [31].

\section{F. Simulation environment and assumptions}

The following assumptions are taken for the study:

- The statistical simulations were carried out on at the center of the band $2.3-2.4 \mathrm{GHz}$ band. In different radio systems simulations the results of the systems would be only in terms of throughput loss or capacity loss, or bit rate loss. To get the interference calculations via the Seamcat tool, the victims and interferes have to be generic. In Monte-Carlo simulations the number of events was 10000 .

- SAP/SAB blocking masks were according to specifications: EN 302064 [32]. The LTE TDD user equipment (MS) according to the ETSI TS 136101 [21] and the base station (BS) according to the ETSI TS 136104 [33]. The reference sensitivities of the LTE BS and MS are defined in the document [16].

- Omni antenna radiation patterns for simulations were generated. Normally horizontal plane gains are between $2 \mathrm{dBi}$ and $10 \mathrm{dBi}$, where gain variations can be realized by reducing the vertical beam width [19] (MS antenna gain $0 \mathrm{dBi}$ and SAP/SAB Tx gain $5 \mathrm{dBi}$ ). Other antennas used were the SEAMCAT tool based. The antenna is according to ITU-R BT.419-3 [34]. The LSA BS used 3-sector $16 \mathrm{dBi}$ antenna gain. Cordless camera and the LSA MS antenna heights were $1.5 \mathrm{~m}$ and the LSA BS antenna height $20 \mathrm{~m}$. The simulations used extended Hata urban below the roof model.

- $\quad$ An LSA (LTE) network with $20 \mathrm{MHz}$ bandwidth and cordless camera $10 \mathrm{MHz}$ bandwidth was used.

- The Seamcat tools based interference criteria was used: minimum carrier-to-interference ratio $(\mathrm{C} / \mathrm{I})=19 \mathrm{~dB}$, $\mathrm{C} /(\mathrm{N}+\mathrm{I})=16 \mathrm{~dB},(\mathrm{~N}+\mathrm{I}) / \mathrm{N}=3 \mathrm{~dB}$ and $\mathrm{I} / \mathrm{N}=0 \mathrm{~dB}$.

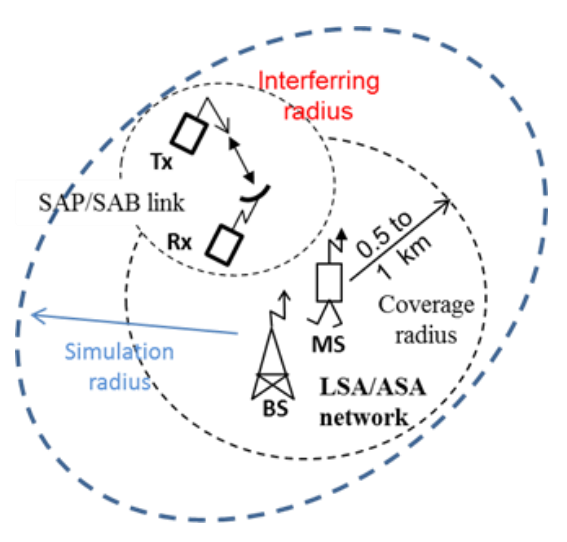

Figure 3 Setup of the simulated system 


\section{G. Simulation procedure}

Simulations environment is depicted in Figure 3, where different coverage radius of the victim and interfering links with respect to the pairs of transmitters and receivers of the victim and interfering links are defined.

Simulation radius indicates various pairs of transmitters and receiver positions used for the simulations. The output power of cordless cameras may vary from $1 \mathrm{~mW}(0 \mathrm{dBm})$ to $1 \mathrm{~W}(30$ $\mathrm{dBm})$ depending on the model and the case. Generally small output powers are used to extend operating time of batterydriven devices and to minimize SAR (Safe Absorption Range). All the transmitters both SAP/SAB and BS and MS in the simulations used low power of $+24 \mathrm{dBm}$.

\section{NUMERICAL RESULTS}

The SEAMCAT tool simulations considering interference on both directions were:

1. LSA (LTE) as interferer (cases a and b)

2. SAP/SAB interferer (cases $c$ and $d$ )

With the LSA (TDD) bandwidth of $20 \mathrm{MHz}$, the system enables an available data rate of up to $150 \mathrm{Mbps}$. Such data speed can deal with simultaneous subscribers in DL and UL, e.g., user average $2 \mathrm{bps} / \mathrm{Hz}$. In TDD, the sub frame can either be DL, UL or a special sub frame between DL and UL period. 3GPP has defined seven uplink-downlink configurations. Data speed definition should be defined by the use in LSA AP.

a) $S A P / S A B$ as victim and MS as interferer

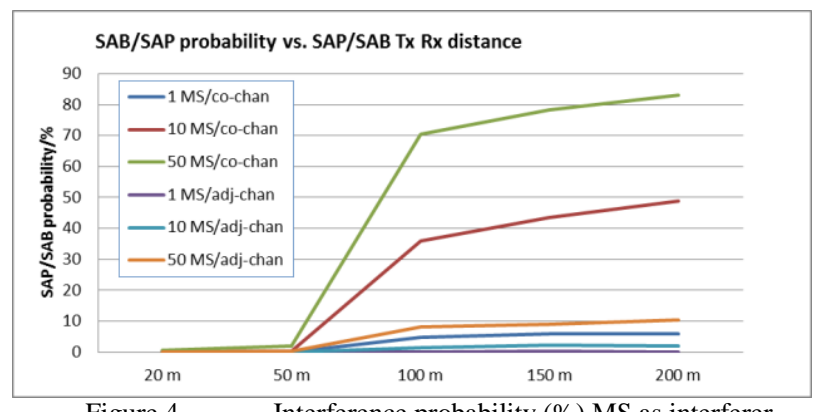

Figure 4 Interference probability (\%) MS as interferer

In this case the SAP/SAB Rx was the victim and the relative position of the interfering $\mathrm{MS}$ to the $\mathrm{SAP} / \mathrm{SAB}$ receiver was selected as constants $(\sim 100 \mathrm{~m})$. The simulation environment (the user defined interferer link radius of MS to BS) was $0.5 \mathrm{~km}$. The sensitivity of the SAP/SAB link is assumed to be $-96 \mathrm{dBm}$. As seen on the figure 4, with these parameters the recommended SAP/SAB Tx and Rx path should be below 50 meters. The results seen in the figure 4 indicate that the more there are active transmitters (MS) the more difficult it is to avoid SAP/SAB Rx probability of interference.

\section{b) $S A P / S A B R x$ as victim and $B S$ as interferer}

In the simulation as BS being the interferer and the $\mathrm{SAP} / \mathrm{SAB} \mathrm{Rx}$ as victim the SAP/SAB Tx and Rx path should be far below 50 meters. The results are seen in the figure 5 .

\section{c) BS as victim and $S A P / S A B$ Tx as interferer}

The local area LSA/LTE application [16/table 20] cell sizes are from 500 to $1000 \mathrm{~m}$. Here the simulated coverage radius between the victim link (BS) receiver and transmitter (MS) was selected variable. The unchecked correlated uncorrelated radius between BS and MS define the range of random movement. To get reasonable results the unchecked value was set to zero. The interference results are shown in the figure 6 with the MS power of 24 and $33 \mathrm{dBm}$. With the tool based interference criterion $(\mathrm{C} / \mathrm{I})$ and the $\mathrm{BS}$ as victim interference probability (\%) is high both in co-channel and adjacent channel simulations.

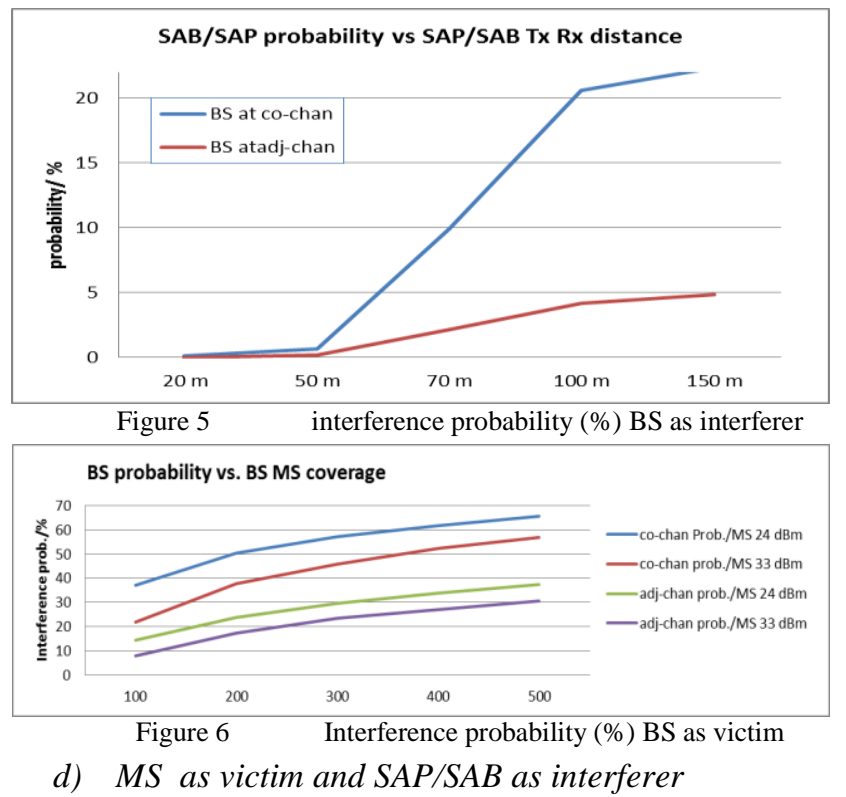

With the same assumptions of the SAP/SAB link as in c simulations were carried out.

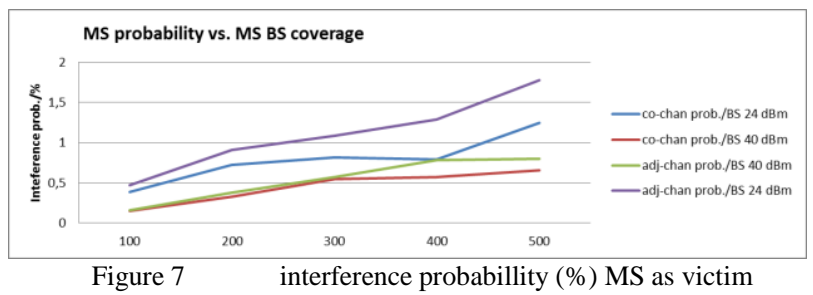

In case of MS as victim the results are seen in the figure 7 and with these parameter MS is more tolerable than the LSA BS.

\section{CONCLUSIONS}

This paper simulated interference compatibility between incumbent programme making and special events (PMSE) and entrant long-term evolution (LTE) mobile communication systems for the new Licensed Shared Access (LSA) concept in the 2.3-2.4 GHz band. Simulation studies were conducted to study the co-channel and adjacent channel interference situations. In the simulations only one incumbent application (cordless camera case) was used. Therefore the results serve as a starting point and it is recommended that both extensive simulations and measurements and internal co-operation in the specific sharing use cases are carried out. Planning rules are required, which define limits of specifications for victims as well as interfering transmitters and receivers performances.

In this study LTE power control was not used. The power control feature is used for capacity purposes to minimize the interference of separate radio links. Also the minimum 
interference ratio should be defined in terms and definitions of ITU quality of service (QoS). From the customer point of view the continuous communication is the requirements. To meet the stringent QoS requirements of real time wideband traffic radio systems also the backhaul network must integrate many of the quality attributes of the core network features.

The environment in simulations was extended Hata urban below the roof model, while most small cell environments are in-door or mixed. As seen from the figures 4-7 of the numerical results the $\mathrm{SAP} / \mathrm{SAB} \mathrm{Rx}$ interference results in certain environment are acceptable as SAP/SAB Tx-Rx distance is low $(\leq 50 \mathrm{~m})$. LSA guard-band designs require extra simulations. Techniques to guarantee proper interference-free operations between systems require also site coordination and site engineering between systems, extra filtering calculations for spurious and adjacent band compatibilities, and in case of TDD, synchronization of the operating base stations as well.

This study focused on sharing between a cordless camera link and LTE network. While seeing the Fig.1, further work is required to describe a model, with which one can conduct extensive simulation environment and parameter values for $\mathrm{CR}$ spectrum sharing capability at the band 2.3-2.4 GHz.

\section{ACKNOWLEDGEMENTS}

This text has been done in close cooperation with CORE+ project.

\section{REFERENCES}

[1] Report ITU-R M.2243. Assessment of the global mobile broadband deployments and forecasts for International Mobile Telecommunications. 2011.

[2] Danijela Cabric, Ian D. O'Donnell, Mike Shuo-Wei Chen, and Robert W. Brodersen, Sharing Radios, IEEE Circuits and Systems Magazine, Second Quarter 2006.

[3] COGEU, Cognitive radio systems for efficient sharing of TV white spaces in European context. Available at: http://www.ict-cogeu.eu/

[4] J. van de Beek, J. Riihijärvi, A. Achtzehn, and P. Mähönen, TV White Space in Europe, IEEE Transactions on Mobile Computing, Vol. 11, No. 2, pp. $178-188$, February 2011.

[5] C. Ghosh, S. Roy, and M. B. Rao, "Modeling and Validation of Channel Idleness and Spectrum Availability for Cognitive Networks," IEEE Journal on Selected Areas in Communications, vol.30, no.10, pp.20292039, November 2012.

[6] J. Zander, L. K. Rasmussen, W. S. Kiwon, P. Mahonen, M. Petrova, R. Jantti, and J. Kronander, On the scalability of cognitive radio: Assessing the commercial viability of secondary spectrum access, IEEE Wireless Communications, Vol. 20, No. 2, 2013.

[7] Rathapon Saruthirathanaworakun, Luis M. Correia, Opportunistic sharing between rotating radar and cellular, IEEE Journal on selected areas in communications, vol. 30, N0. 10 November 2012, pp. $1900-$ 1910.

[8] ECC's Frequency Management Working Group (WG FM), "Report on ASA concept," FM(12)084 Annex 47, 2012. Available at: http://www.cept.org//files/8694/FM(12)084\%20Annex\%2047_Report\% 20on\%20ASA\%20concept.doc

[9] M. Matinmikko et. al., Cognitive Radio Trial Environment, First Live Authorized Shared Access-Based Spectrum-Sharing Demonstration, IEEE Vehicular Technology Magazine, September 2013.

[10] The President's Council of Advisors on Science and Technology (PCAST) Report, Executive Office of the President President's Council of Advisors on Science and Technology, July 2012
[11] FCC workshop material, January 2014. Available at: http://wireless.fcc.gov/workshops/sas_01-14-2014/end/SandersNTIA.pdf

[12] E. Perez, K.-J. Friederichs, A. Lobinger, I. Viering and J. D. Naranjo, Optimization of Authorised/Licensed Shared Access resources. CrownCom 2014, pp. 241-246.

[13] Report on Collective Use of Spectrum (CUS) and other spectrum sharing approaches. RSPG11-392, Radio Spectrum Policy Group, Nov. 2011.

[14] RSPG Opinion on Licensed Shared Access. RSPG13-538, Radio Spectrum Policy Group, November 2013.

[15] ECC Report 205. Licensed Shared Access (LSA). February 2014.

[16] Electromagnetic compatibility and radio spectrum matters (ERM) system reference document (SRdoc) mobile broadband services in the $2300-2400 \mathrm{MHz}$ frequency band under licensed shared access regime, ETSI TR103113(2013-07). Available at: http://www.etsi.org/deliver/etsi_tr/103100_103199/103113/01.01.01_60/ tr 103113v010101p.pdf

[17] Claus Hetting \& Stefan Stanislawski, LTE TDD: The preferred choice for mobile broadband in unpaired bands Ventura Team LLP, 03. 2010

[18] Acceleran white paper, The essential importance of LTE TDD for small cell deployments, July $2013 . \quad$ Available at: www.acceleran.com/sites/default/files/AcceleranWP101 0.pdf

[19] ERC Report 38, Handbook on Radio equipment and video links for ENG/OB use

[20] ECC Report 172, Broadband wireless systems usage in the 2300-2400 $\mathrm{MHz}$

[21] 3GPP TS 36.101, 3rd Generation Partnership Project;Technical Specification Group Radio Access Network;Evolved Universal Terrestrial Radio Access (E-UTRA);User Equipment (UE) radio transmission and reception (Release 10) V10.6.0 (2012-03)

[22] Recommendation ITU-R SM.1599, "Deteminination of the geographic and frequency distribution of the spectrum utilization factor for frequency planning purposes"

[23] Recommendation ITU-R SM.1046-2 (05/2006), "Definition of spectrum use and efficiency of a radio system"

[24] Youping Zhao, James O. Neel, Performance Evaluation of Cognitive Radios: Metrics, Utility Functions, and Methodology, Proceedings of the IEEE, vol. 97, No 4 April 2009

[25] Welch J., Clark J., A proposed media delivery index (MDI), IETF Request for comments: 4445, April 2006

[26] ERC25, The European Table of Frequency Allocations and Applications in the Frequency Range $8.3 \mathrm{kHz}$ to $3000 \mathrm{GHz}$ (ECA TABLE), approved February 2013

[27] ECC working group FM52 and FM53. Available at http://www.cept.org/ecc/groups/ecc/wg-fm/fm-52 and http://www.cept.org/ecc/groups/ecc/wg-fm/fm-53

[28] Z.A. Shamsan, A.M. Al-Hetar, T. Abd. Rahman, Spectrum Shearing of IMT-Advanced and FWA Services under different Clutter Loss and Bandwidth Effects. Progress in Electromagnetics Research, PIER 87, pp. 331-344, 2008

[29] ERC Report 68, Monte Carlo simulation methodology for the use of sharing and compatibility studies between different radio services or systems.

[30] SEAMCAT 4.1.0, the Monte-Carlo Radio simulation tool. The SW can be contacted via email: ero@ero.dk .

[31] The Seamcat Handbook, January 2010, European Communication Office

[32] ETSI EN 302064, Electro-magnetic compatibility and radio spectrum matters (ERM), Wireless Video Links (WVL) operating in the 1.3 to 50 $\mathrm{GHz}$ frequency band

[33] 3GPP TS 36.104, LTE; Evolved Universal Terrestrial Radio Access (EUTRA); Base Station (BS) radio transmission and reception (3GPP TS 36.104 version 11.5.0 Release 11)

[34] ITU-R BT.419-3, Directivity and polarization discrimination of antennas in the reception of television broadcastin 